\section{Planificación de la preparación para la influenza pandémica: esfuerzos regionales ${ }^{1}$}

\author{
Oscar J. Mujica, ${ }^{2}$ Otávio Oliva, ${ }^{2}$ \\ Thais dos Santos ${ }^{2}$ \\ y John P. Ehrenberg ${ }^{2}$
}

Forma de citar: Mujica OJ, Oliva O, Santos T, Ehrenberg JP. Planificación de la preparación para la influenza pandémica: esfuerzos regionales. Rev Panam Salud Publica. 2008;23(6):428-34.

Palabras clave: gripe aviar, gripe humana, brotes de enfermedades, preparación, Américas.

\footnotetext{
Traducción de: Mujica OJ, Oliva O, dos Santos T, Ehrenberg JP. Pandemic influenza preparedness: regional planning efforts. En: Institute of Medicine. Ethical and legal considerations in mitigating pandemic disease. Workshop summary. Washington, D.C.: National Academies Press; 2007. Todos los derechos reservados. Publicado con permiso expreso de National Academies Press y la autorización de la Academia Nacional de Ciencias de los Estados Unidos de América.

2 Equipo de Influenza Pandémica, Unidad de Enfermedades Transmisibles, Organización Panamericana de la Salud, Washington, D.C., Estados Unidos de América. La correspondencia se debe enviar a Oscar J Mújica, Área de Vigilancia Sanitaria y Atención de Enfermedades, Organización Panamericana de la Salud, 525 Twenty-third St. NW, Washington, D.C., 20037, USA. Correo electrónico: mujicaos@ paho.org

3 Institute of Medicine. Ethical and legal considerations in mitigating pandemic disease. Workshop summary. Washington, D.C.: National Academies Press; 2007.
}

\section{Nota de la Redacción}

La detección de casos humanos de influenza aviar H5N1 en Asia y África ha centrado la atención mundial debido a que este virus no había afectado a seres humanos con anterioridad. Hasta el 30 de abril de 2008, la Organización Mundial de la Salud (OMS) había notificado 382 casos confirmados de personas infectadas por la cepa H5N1 del virus de la influenza A, con una tasa de letalidad superior a 60\%. La emergencia de una nueva cepa del virus de la influenza aviar capaz de infectar a los humanos, aunada a la falta de exposición anterior al virus -y por ende una elevada tasa de susceptibilidad - despierta la preocupación de que la enfermedad pueda propagarse rápidamente y dar lugar a una pandemia.

Con frecuencia se comparan los daños que podría provocar una posible pandemia de influenza con los efectos ocasionados por los desastres naturales, aunque se anticipa que los resultados de tal pandemia serían más generales y continuos y, por consiguiente, podrían agotar los recursos de los países. La persistencia de este virus y las posibilidades de que provoque una pandemia ponen de relieve la necesidad de que todos los países elaboren planes nacionales de preparación ante una epidemia de influenza y los pongan en práctica.

En virtud de este riesgo global, la Organización Panamericana de la Salud (OPS) ha priorizado la cooperación con sus Estados Miembros en la formulación de los planes nacionales de preparación para enfrentar una pandemia de influenza. El objetivo de la cooperación técnica es que los países cuenten con planes nacionales intersectoriales y operativos y que estos se apliquen a niveles nacional, intermedio y local. Paralelamente a la planificación, se ha apoyado a los países en la generación, el fortalecimiento y el mantenimiento de sus capacidades básicas de alerta y respuesta —según se establece en el nuevo Reglamento Sanitario Internacional adoptado en 2005-, a fin de controlar oportunamente una posible situación pandémica y mitigar los efectos de cualquier otro evento de salud pública de importancia internacional.

La promoción de planes nacionales integrales e intersectoriales se ha reforzado con la realización de talleres multidisciplinarios diseñados para que cada país evalúe su plan nacional. A invitación del Instituto de Medicina de las Academias Nacionales de los Estados Unidos de América, los resultados de esas evaluaciones se presentaron en el Taller sobre Consideraciones Éticas y Legales en la Atenuación de Enfermedades Pandémicas (Ethical and Legal Considerations in Mitigating Pandemic Disease), organizado en Washington, D.C., por el Foro sobre Amenazas Microbianas del Consejo de Salud Global.

Dada la trascendencia actual del tema y la importancia de diseminar esta información entre la mayor audiencia de habla hispana en las Américas, la Revista Panamericana de Salud Pública / Pan American Journal of Public Health publica a continuación la traducción íntegra al español de este documento. ${ }^{3}$ Esperamos que su publicación contribuya a ampliar el conocimiento sobre el tema y a promover la preparación necesaria en los países de la Región con vistas a enfrentar una posible pandemia de influenza. 
"Ver después no vale: lo que vale es ver antes... y estar preparados." José Martí

En 2003, la 56. ${ }^{\text {a }}$ Asamblea Mundial de Salud y el $44 .^{\circ}$ Consejo Directivo de la Organización Panamericana de la Salud (OPS) emitieron resoluciones que urgían a los países a fortalecer su capacidad para prevenir, detectar y diagnosticar la infección por el virus de la influenza y a estar preparados para responder a una situación pandémica (1-2). Con el fin de evitar las consecuencias catastróficas que podrían acompañar a una pandemia de influenza a escala mundial, tales planes de contingencia deben ponerse en práctica ahora, durante el período interpandémico, sin esperar por la ocurrencia de la próxima pandemia.

En 2006, cerca de 130 millones de personas - es decir, 23\% de la población de América Latina y el Caribe- vivían en áreas rurales de América Latina y el Caribe (3), la mayoría de ellas en contacto directo con pollos y cerdos. La Organización de las Naciones Unidas para la Agricultura y la Alimentación (FAO) informó que a las aves de corral corresponde cerca de $70 \%$ de la proteína animal consumida en América Latina y el Caribe (4). Además, la creciente industria avícola se ha convertido en una importante fuente de ingresos y de empleo en estos países, y contribuye en gran parte a su desarrollo urbano y periurbano. Una pandemia en la Región no sólo constituiría un problema de salud pública, sino también una amenaza a la seguridad alimentaria y un desastre económico para las poblaciones rurales más pobres y las economías nacionales en su conjunto.

Considerando la amenaza que plantea una posible pandemia de influenza, la OPS ha venido apoyando a sus Estados Miembros en la preparación para enfrentar una pandemia, de acuerdo con el mandato emanado de sus cuerpos directivos y la Cumbre de las Américas de 2005. Ese año, la Directora de la OPS creó el Grupo de Trabajo sobre Alerta y Respuesta Epidémicas como equipo multidisciplinario encargado de asesorar, coordinar y monitorear todas las actividades de la Organización relacionadas con la planificación y puesta en marcha de la preparación y respuesta frente a una pandemia de influenza. Todas las actividades de este grupo de trabajo se rigen por el Reglamento Sanitario Internacional (RSI), revisado, adoptado en mayo de 2005 (5). Este reglamento estipula que los países desarrollen y mantengan capacidades básicas para detectar, evaluar y controlar eventos de importancia para la salud pública internacional. La natura- leza interprogramática del grupo de trabajo mencionado le permite abordar mejor el complejo proceso relacionado con la aplicación del RSI y la planificación para una pandemia de influenza, lo cual demanda esfuerzos altamente coordinados desde diversos sectores.

Hasta el momento, la cooperación técnica de la OPS se ha centrado en el apoyo a los Estados Miembros en la formulación de planes nacionales de preparación para una pandemia de influenza (PNPPI) - tomando en cuenta las recomendaciones que ofrece el Plan Global de Preparación para la Influenza, de la OMS - con vistas a establecer medidas nacionales antes y durante las pandemias (6). En el cuadro 1 se resumen los pasos clave en el desarrollo y la evaluación de los PNPPI.

CUADRO 1. Estrategia de la Organización Panamericana de la Salud en apoyo a los Estados Miembros para el desarrollo y la evaluación de los planes nacionales de preparación para una pandemia de influenza (PNPPI)

1. Elaboración de los borradores de los PNPPI

a. Presentación de las Guías de la OMS sobre la planificación de la preparación para una pandemia

b. Presentación y aplicación de herramientas de modelación, tales como FluAid, FluSurge y FluWorkLoss, a fin de estimar el posible impacto de una pandemia

c. Desarrollo de planes de acción nacionales, incluida la formulación adecuada de borradores de PNPPI

2. Evaluación y puesta a prueba de los borradores de los PNPPI

a. Autoevaluación de los PNPPI

b. Realización de ejercicios de gabinete para detectar problemas en la cadena de mando y necesidades de integración y coordinación multisectoriales

c. Desarrollo de planes de acción para identificar brechas remanentes detectadas durante los ejercicios de autoevaluación y de simulación

3. Aplicación de los PNPPI a nivel local

a. Desarrollo de entrenamientos de simulación y ejercicios de gabinete para comprobar la preparación local y poner en práctica los planes locales de contingencia

b. Promoción de entrenamientos multisectoriales a nivel local para promover el desarrollo de planes locales de contingencia para una pandemia que incorporen adecuadamente todas las áreas pertinentes, entre ellas la vigilancia, los servicios de salud, el control de desastres y la comunicación social

c. Realización de ejercicios de gabinete para comprobar la integridad de los planes locales, tomando en consideración las realidades específicas dentro de los países

4. Monitoreo y fortalecimiento de los PNPPI

a. Promoción del uso de simulacros locales dentro del país para apoyar el monitoreo de la pertinencia de los planes locales de contingencia

b. Promoción de los cambios necesarios para mantener los planes actualizados

Fuente: Organización Panamericana de la Salud (7). 
CUADRO 2. Impacto potencial de una pandemia de influenza con una tasa de ataque clínico de $25 \%$ en América Latina y el Caribe, según los principales criterios de desenlace y escenarios de gravedad, a mediados de 2006

\begin{tabular}{lcc}
\hline & \multicolumn{2}{c}{ Escenario pandémico } \\
\cline { 2 - 3 } $\begin{array}{c}\text { Impacto potencial } \\
\text { en la salud }\end{array}$ & Moderado & 1918 \\
\hline Defunciones & $334163[131630-654960]$ & $2418469[627367-5401035]$ \\
Hospitalizaciones & $1461401[459051-1937503]$ & $11798613[3189747-16418254]$ \\
Consultas ambulatorias & $76187593[59738730-109207769]$ & $68470386[58114124-92227761]$ \\
\hline
\end{tabular}

Fuente: Organización Panamericana de la Salud (7).

Los planes multisectoriales deben tomar en consideración tanto la salud humana como la animal y deben ser lo suficientemente flexibles para tener en cuenta varios posibles escenarios de una pandemia, en dependencia de los niveles de patogenicidad viral y la disponibilidad de recursos. Se han llevado a cabo talleres subregionales para entrenar en el uso de paquetes estadísticos de modelación a los encargados de elaborar los PNPPI. Estas herramientas de modelación han sido desarrolladas por los Centros para el Control y la Prevención de Enfermedades de los Estados Unidos de América (CDC) para estimar el impacto potencial de una pandemia (8-10). La disponibilidad de tales estimados ayudará a los países a mantener sus planes nacionales flexibles, ya que ofrecen una gama de contingencias para las que se requiere planificación —entre ellas el peor escenario posible, en el que no hay ni vacunas ni medicamentos antivirales disponibles-. En el cuadro 2 se muestra un resumen de los estimados del impacto de una posible pandemia de influenza en América Latina y el Caribe con escenarios similares a los de 1968 y 1918, estimados preparados por los equipos de los países durante los talleres subregionales mencionados.

Después que los Países Miembros desarrollaron los borradores de sus planes, la OPS realizó una serie de ejercicios de autoevaluación en los que se analizaron los PNPPI mediante una herramienta desarrollada por la OPS, basada en la lista de verificación de la OMS, para la planificación de la preparación para una pandemia de influenza (11). Esa herramienta abarca los siete componentes básicos de la lista de verificación de la OMS: la preparación ante una emergencia; la vigilancia; la investigación y el tratamiento de casos; la contención de la población; la preservación de los servicios básicos; la investigación y la evaluación; y la aplicación, la puesta a prueba y la revisión del plan nacional. Estos componentes básicos se subdividen, a su vez, en 44 categorías principales que contienen un total de 368 puntos de verificación para la evaluación.
Todos los países de América Latina y el Caribe participaron en esos ejercicios de autoevaluación. La delegación de cada país contó con participantes de las áreas de epidemiología, servicios de salud, diagnóstico de laboratorio, vacunación, control de desastres, preparación para emergencias, comunicación social, salud pública veterinaria, agricultura y relaciones internacionales. El propósito principal de los ejercicios fue propiciar el trabajo conjunto de los países de un modo colectivo, multidisciplinario e intersectorial para mejorar la preparación y la aplicación de sus diversos planes nacionales. Para muchos de estos profesionales, este ejercicio constituyó la primera oportunidad de sentarse con sus colegas para analizar sobre la mesa la preparación ante una pandemia. Uno de los logros principales de esa interacción fue la discusión multisectorial que tuvo lugar a propósito de los pasos requeridos para completar los planes nacionales de los distintos países. En particular, la discusión resaltó la importancia del trabajo articulado y la integración en el proceso de planificación de una contingencia pandémica.

Los planes nacionales de los países participantes mostraron diferentes grados de observancia de la lista de verificación de la OMS (11), y las diversas subregiones tendieron a presentar patrones diferentes de fortalezas y debilidades en sus niveles de observancia. ${ }^{4}$ En los países andinos, por ejemplo, los planes nacionales parecían ser más abarcadores en los aspectos relacionados con el manejo de los casos, mientras que las áreas de investigación y evaluación no parecían estar tan bien. Los países del

\footnotetext{
4 Se evaluaron cuatro subregiones: el Área Andina, compuesta por Bolivia, Colombia, Ecuador, Perú y Venezuela; el Cono Sur, compuesto por Argentina, Brasil, Chile, Paraguay y Uruguay; América Central, compuesta por Costa Rica, El Salvador, Guatemala, Honduras, Nicaragua y Panamá (más Cuba, Haití y la República Dominicana); y el Caribe, compuesto por Anguila, Antigua y Barbuda, Aruba, las Bahamas, Barbados, Belice, Bermuda, Dominica, Granada, Guyana, Islas Caimán, Isla de San Martín, Islas Turcas y Caicos, Islas Vírgenes Británicas, Jamaica, Martinica, Montserrat, Saint Kitts y Nevis, Santa Lucía, San Vicente y las Granadinas, Suriname, y Trinidad y Tobago.
} 
CUADRO 3. Disposición de la preparación para una pandemia en América Latina y el Caribe. Grado de cumplimiento actual (porcentaje) de las pautas de la Organización Mundial de la Salud, según los componentes básicos y las subregiones de la Organización Panamericana de la Salud, a mediados de 2006

\begin{tabular}{|c|c|c|c|c|}
\hline Componente básico del $\mathrm{PNPPI}^{\mathrm{a}}$ & Área Andina & América Central & Caribe & Cono Sur \\
\hline 1. Preparación para situaciones de emergencia & 38,6 & 34,6 & 56,7 & 58,5 \\
\hline 2. Vigilancia epidemiológica & 37,0 & 34,8 & 56,5 & 54,4 \\
\hline 3. Manejo de casos & 52,3 & 54,5 & 48,9 & 60,9 \\
\hline 4. Contención de la población & 20,0 & 38,0 & 37,0 & 64,0 \\
\hline 5. Continuidad de los servicios esenciales & 24,5 & 33,3 & 45,2 & 41,9 \\
\hline 6. Investigación y evaluación & 10,0 & 40,0 & 15,0 & 30,2 \\
\hline 7. Puesta en práctica del plan nacional & 40,0 & 60,0 & 30,0 & 50,0 \\
\hline
\end{tabular}

Fuente: Organización Panamericana de la Salud (7).

a PNPPI: plan nacional de preparación para una pandemia de influenza.

CUADRO 4. Cuestiones fundamentales legales y éticas a considerar y evaluar en los planes nacionales de preparación para una pandemia de influenza

\section{Cuestiones legales}

1. Constatación de un marco legislativo vigente para el plan de respuesta nacional

2. Disposiciones legales para casos imprevistos (preservación de los servicios básicos y otras medidas de control de crisis)

3. Evaluada la base legal sobre las restricciones de movimientos $y$ viajes

4. Evaluada la base legal sobre el cierre de centros educativos

5. Evaluada la base legal para el aislamiento y la cuarentena de personas infectadas o sospechadas de estar infectadas

6. Evaluada la base legal para la prohibición de reuniones masivas

7. Evaluadas las políticas vigentes y las bases legales para la vacunación del personal imprescindible

8. Evaluados los aspectos legales (responsabilidad legal, aseguramiento y licencias) relacionados con la movilización de trabajadores temporales

9. Considerada la responsabilidad legal por efectos adversos imprevistos atribuidos a vacunas específicas y medicamentos antivirales usados contra la pandemia

10. En vigor el marco legislativo para cumplir con el Reglamento Sanitario Internacional

11. Considerada la inclusión de la influenza pandémica en la legislación nacional para la prevención de enfermedades ocupacionales

\section{Cuestiones éticas}

1. Revisión ética sobre la limitación/restricción del acceso a recursos escasos

2. Revisión ética sobre el carácter obligatorio de la vacunación del personal imprescindible

3. Revisión ética sobre la limitación de la libertad y el movimiento de las personas, como lo que pudiera ocurrir durante el aislamiento y la cuarentena

4. Establecimiento de un marco ético para la investigación, en especial cuando incluya seres humanos

Fuente: Organización Mundial de la Salud (11).

Caribe también parecían tener dificultades con el área de investigación y evaluación, pero estaban fuertes en el área de preparación para enfrentar una emergencia. Los planes nacionales de los países centroamericanos resultaron los más fuertes en su puesta en práctica y los más débiles en la continuidad de los servicios esenciales. En los países del Cono Sur, el desarrollo del componente de contención poblacional fue una fortaleza y la continuidad de los servicios esenciales fue una debilidad. En el cuadro 3 se presenta el grado de cumplimiento promedio de las guías de la OMS en las cuatro subregiones geográficas, según cada uno de los siete componentes básicos de los PNPPI.

La preparación para situaciones de emergencia, como primer componente básico de la planificación de la preparación para enfrentar una pandemia de influenza, está formada básicamente por seis categorías principales. Una de ellas está dedicada a cuestiones específicas y fundamentales tanto legales como éticas, como se describe en el cuadro 4.

De todas las categorías de preparación para situaciones de emergencia, la de aspectos legales y éticos es en la que los países latinoamericanos necesitan trabajar más para cumplir con la lista de verificación de la OMS ya mencionada. Esta, como se puede observar en el cuadro 5, está por mucho entre las áreas menos desarrolladas en los países andinos y centroamericanos y es la segunda área menos desarrollada en las otras dos subregiones.

En los talleres se subrayó la necesidad de coordinación intersectorial en el desarrollo de los PNPPI. Desde febrero de 2006, cuando comenzaron los talleres, muchos países han estado inmersos en el diálogo intersectorial, además de llevar a cabo actividades necesarias para la aplicación a nivel local de los PNPPI. A partir del impulso generado por la sesión subregional de autoevaluación, los profesionales encargados de planificar la preparación han fortalecido su interacción con los que serán responsables de aplicar los planes. La discusión resultó en una mejor definición de las funciones y responsabilidades de todos los actores involucrados en la respuesta. También fue extremadamente beneficioso que los repre- 
CUADRO 5. Evaluación del componente de preparación para emergencias en los planes nacionales de preparación para una pandemia de influenza, que abarca aspectos legales y éticos, en América Latina y el Caribe. Cumplimiento actual (porcentaje) de las pautas de la Organización Mundial de la Salud, según las áreas principales y las subregiones de la Organización Panamericana de la Salud, a mediados de 2006

\begin{tabular}{|c|c|c|c|c|}
\hline Preparación para situaciones de emergencia & Área Andina & América Central & Caribe & Cono Sur \\
\hline Movilización política & 58,3 & 55,6 & 66,7 & 88,9 \\
\hline Dirección y control & 37,5 & 22,2 & 58,3 & 66,7 \\
\hline Evaluación de riesgos & 40,0 & 50,0 & 37,5 & 40,0 \\
\hline Comunicación de riesgos & 38,5 & 41,7 & 62,5 & 75,0 \\
\hline Aspectos legales y éticos & 11,1 & 13,3 & 40,0 & 60,0 \\
\hline Plan de respuesta por fases & 33,3 & 33,3 & 66,7 & 100,0 \\
\hline
\end{tabular}

Fuente: Organización Panamericana de la Salud (7).

sentantes de los departamentos jurídicos de los ministerios de salud participaran en el proceso, porque pudieron indicar las enmiendas a la legislación sanitaria que serían necesarias para aplicar los PNPPI.

Además de promover el desarrollo de los planes nacionales, la OPS está ayudando a sus Estados Miembros a que fortalezcan los mecanismos y las capacidades necesarios para la plena aplicación de estos planes. Entre las capacidades necesarias se encuentran las de vigilancia, servicios de salud, tecnologías de vacunas y antivirales, y comunicación, entre otras. En particular, la OPS está apoyando a sus Estados Miembros para hacer los PNPPI operativos a nivel local, ya que los planes nacionales de preparación para enfrentar la influenza son solamente tan eficaces como lo sean sus planes locales de contingencia. Una meta importante será fortalecer las competencias básicas de los Estados Miembros y de las comunidades para responder a cualquier emergencia de salud pública, según se identifica a través del nuevo RSI.

Históricamente, las pandemias de influenza han tomado al mundo por sorpresa, al dejar un tiempo mínimo para que los servicios de salud se preparen ante el rápido incremento en el número de casos y defunciones que caracteriza estos eventos y que los hace tan dañinos (12). Sin embargo, la situación actual es marcadamente diferente, ya que el mundo ha sido advertido con anticipación. Esta advertencia por adelantado brinda una oportunidad sin precedentes, especialmente en las Américas, para prepararse para una pandemia y desarrollar formas de atenuar sus efectos, aun en áreas con problemas de acceso a los servicios básicos de salud.

La información científica disponible indica que una pandemia de influenza se sentirá más intensamente a nivel de la comunidad, especialmente en los jóvenes, los pobres y otros grupos vulnerables (13). A pesar de los extraordinarios avances logrados en el aumento de la preparación para una pandemia de influenza a nivel de países, todavía queda un considerable desafío para lograr implementar la preparación a nivel local dentro de los países - con los encargados de tomar las decisiones, los médicos y los ciudadanos involucrados, que estarán encargados de la aplicación real de los planes nacionales- En la medida en que se establecen las estrategias nacionales, el enfoque de la OPS se está desplazando de la planificación y la sensibilización al incremento de la aceptación local y la adopción de estas estrategias a ese nivel, así como a asegurar su aplicación eficaz. Para cerrar la brecha existente entre la planificación y la puesta en práctica, se debe motivar a quienes estén ejecutando los planes a nivel local para que participen en el proceso de planificación nacional. La aplicación local de los PNPPI debe someterse a pruebas mediante simulaciones y ejercicios de gabinete con el fin de comprobar los planes locales de preparación y contingencia. Además, la planificación de la contingencia a nivel local se debe promover y apoyar mediante una medida más amplia: mejorar la capacidad de los Estados Miembros y las comunidades para responder a todo tipo de emergencias de salud pública y no solo a la influenza pandémica.

La Región de las Américas está en una posición afortunada al tener la oportunidad de prepararse para una pandemia de influenza antes de que el virus entre en esta parte del mundo. Sin embargo, el hecho de que la Región no se haya visto afectada hasta ahora crea un falso sentido de seguridad y hace que no parezca tan urgente la preparación para una pandemia. El resultado es que la preparación para una pandemia parece menos importante en comparación con muchas otras prioridades concurrentes y hace, por tanto, que figure menos en las agendas políticas de muchos de los países.

Todos los preparativos para una pandemia se deben llevar a cabo dentro del marco proporcionado por el RSI revisado, el cual establece un primer nivel de competencias básicas que los países 
CUADRO 6. Logros de los Estados Miembros de la Organización Panamericana de la Salud en el desarrollo y evaluación de los planes nacionales de preparación para una pandemia de influenza (PNPPI)

- Profesionales de varios sectores que colaboran, a menudo por primera vez, en la formación de capacidades nacionales para hacer frente a una pandemia

- Los países están creando, analizando y ajustando sus PNPPI de una manera integrada y coordinada

- Inclusión de la preparación para una pandemia de influenza en los Programas de Acción Sanitaria de los Sistemas Regionales de Integración (MERCOSUR, CARICOM, Comunidad Andina, Secretaría de Integración Social Centroamericana)

- Fortalecimiento básico de la infraestructura de salud pública, orientado hacia una posible pandemia de influenza, pero también aplicable a una amplia gama de emergencias de salud pública

- Grupo regional de profesionales capacitados en múltiples aspectos de la preparación para la influenza - prestación de servicios de salud, vigilancia, comunicación social y de riesgo, y control de desastres y emergencias

- Profesionales que pueden reproducir los entrenamientos para colaboradores y colegas a nivel local en sus países

- Compromiso de los profesionales capacitados para proseguir con actividades de preparación para la influenza

Fuente: Organización Panamericana de la Salud (7).

deben alcanzar para detectar y responder a cualquier emergencia de salud pública de importancia internacional. De manera análoga, las estructuras y los mecanismos existentes, tales como los planes de contingencia para la atenuación de emergencias y desastres nacionales, se deben usar en la preparación para enfrentar la influenza pandémica a fin de evitar la duplicación de esfuerzos y optimizar la utilización de los recursos disponibles.

La OPS, con una historia de 105 años de colaboración con los países de las Américas, ha creado una sólida base que se puede utilizar en la preparación para enfrentar una posible pandemia de influenza. A partir de su experiencia en el apoyo a los Estados Miembros de América Latina y el Caribe, la OPS puede ser un socio eficaz que ayude a desarrollar y revisar los PNPPI, así como a consolidar sus logros actuales (cuadro 6).

Las amenazas mundiales actuales, entre ellas la influenza pandémica, requieren de un esfuerzo concertado de todos los que estén en capacidad de emprender una acción eficaz. La OPS reconoce la importancia primordial de las asociaciones en ese esfuerzo y está colaborando estrechamente con varios socios interesados en el tema. También seguirá promoviendo los enfoques multisectoriales y multinacionales - como los empleados durante los ejercicios de planificación - para fortalecer la cooperación, la vigilancia y la comunicación.
Claramente, se necesitarán recursos adicionales para alcanzar las múltiples metas, por ejemplo que varios países promuevan el apoyo de las contrapartes, que se aprovechen los sistemas existentes de vigilancia y que estos se amplíen para convertirse en sistemas de base poblacional, y que se amplíe el nivel de preparación y las capacidades de respuesta rápida hasta el nivel local. Además, el acceso a los medicamentos, las vacunas y otros suministros es todavía un problema no resuelto. No obstante, creemos que la Región tiene el potencial para ser autosuficiente. Un reto final será extender la participación y el compromiso intersectoriales al sector privado, las organizaciones no gubernamentales (ONG) y los sectores académicos. Resta aún formular mecanismos para que esto se lleve a cabo.

En conclusión, la amenaza de una pandemia de influenza ha revelado las debilidades de algunos sistemas en las Américas, pero también ha demostrado una vez más la fuerte determinación de los países de la Región a colaborar juntos, trabajar con rapidez, superar las disparidades y compartir la información. La cooperación técnica ha servido para fortalecer la salud pública en estos países. Esto constituye una contribución mundial importante y a la postre podría salvar muchas vidas.

\section{SYNOPSIS}

\section{Preparedness planning for an influenza pandemic: Regional efforts}

Failure to establish a contingency plan prior to a public health emergency can have catastrophic consequences. The threat of a new influenza pandemic has prompted countries to draft national strategic preparedness plans to prevent, mitigate, and recover from a potential influenza pandemic. This paper examines these preparations in Latin America and the Caribbean and describes potential scenarios of pandemic impact on the burden of mortality and on health services in the Region. In particular, the paper reports on the progress made by Member States in developing national influenza pandemic preparedness plans and implementation mechanisms at both the national and local levels. These achievements were facilitated through a series of planning workshops and selfassessment exercises conducted by PAHO for intersectoral country teams and guided by the WHO global influenza preparedness plan. Although significant progress has been made in plan completeness, intercountry preparedness planning and local level implementation remain key challenges. Multisectoral partnerships are clearly paramount to securing the commitment and resources needed to reach and sustain effective pandemic preparedness in the Americas.

Key words: influenza in birds; influenza, human; disease outbreaks; preparedness; Americas. 


\section{REFERENCIAS}

1. World Health Organization. Resolution WHA56.19. Prevention and control of influenza pandemics and annual epidemics. 56. World Health Assembly. Geneva: WHO; 2003. Hallado en: http://www.who.int/gb/e/e_wha56. $\mathrm{html}$ Resolutions. Acceso el 15 de enero de 2007.

2. Pan American Health Organization. Resolution CD44.R8. Influenza pandemic: preparation in the Hemisphere. 44. ${ }^{\circ}$ Directing Council. Washington, D.C.; PAHO; 2003. Hallado en: http:// www.paho.org/english/gov/cd/cd44 index-e.htm\#Resolutions. Acceso el 15 de enero de 2007.

3. United Nations. World urbanization prospects: the 2005 revision. New York: Department of Economic and Social Affairs; 2006. Hallado en: http:/ /www.un. org/esa/population/publications / WUP2005/2005wup.htm. Acceso el 17 de enero de 2007. (ESA/P/WP/200.)

4. Food and Agriculture Organization. Helping prevent avian influenza in Latin America and the Caribbean. FAONewsRoom [sitio en Internet]. Roma: FAO; 2006. Hallado en: http:// www.fao.org/ newsroom/en/news/2006/1000381/ index.html. Acceso el 11 de enero de 2007.

5. World Health Organization. Resolution WHA58.3. Revision of the International
Health Regulations. 58..$^{\circ}$ World Health Asembly. Geneva: WHO; 2005. Hallado en: http://www.who.int/gb/e/e_wha 58.html/\#Resolutions. Acceso el 15 de enero de 2007.

6. World Health Organization. Global Influenza Preparedness Plan: the role of WHO and recommendations for national measures before and during pandemics. Global Influenza Programme. Geneva: WHO; 2005. Hallado en: http://www.who.int/csr/resources/ publications/influenza/WHO_CDS CSR_GIP_2005_5/en/index.html. Acceso el 16 de enero de 2007. (WHO/ CDS/CSR/ GIP/2005.5.)

7. Pan American Health Organization. Health in the Americas. 2007 ed. Vol. I. Washington, D.C.: PAHO; 2007.

8. Meltzer MI, Shoemake HA, Kownaski M, Crosby R. FluAid 2.0: software and manual to aid state and local-level health officials plan, prepare and practice for the next influenza pandemic (beta test version). Atlanta: Centers for Disease Control and Prevention; 2002. Hallado en: http://www.cdc.gov/flu/ tools/fluaid/. Acceso el $1 .^{\circ}$ de mayo de 2007.

9. Zhang X, Meltzer MI, Wortley P. FluSurge 2.0: software and manual to aid state and local public health officials and hospital administrators in estimating the impact of an influenza pandemic on hospital surge capacity (beta test version). Atlanta: Centers for Disease Control and Prevention; 2005. Hallado en: http://www.cdc.gov/flu/tools/flu surge/. Acceso el 1. ${ }^{\circ}$ de mayo de 2007.

10. Praveen D, Zhang $X$, Meltzer MI Bridges CB. FluWorkLoss 1.0: software and manual to aid state and local public health officials estimating the impact of an influenza pandemic on work day loss (beta test version). Atlanta: Centers for Disease Control and Prevention; 2006. Hallado en: http://www.cdc.gov/flu/ tools/fluworkloss/. Acceso el $1 .^{\circ}$ mayo de 2007.

11. World Health Organization. Checklist for influenza pandemic preparedness planning. Geneva: WHO; 2005. Hallado en: http://www.who.int/csr/resources/ publications/influenza/WHO_CDS CSR_GIP_2005_4/en/index.html. Acceso el 16 de enero de 2007.

12. Glezen WP. Emerging infections: pandemic influenza. Epidemiol Rev. 1996; 18(1):64-76.

13. Institute of Medicine. The threat of pandemic influenza: are we ready? Washington, D.C.: National Academies Press; 2005.

\section{El control de las enfermedades transmisibles, 18a. edición}

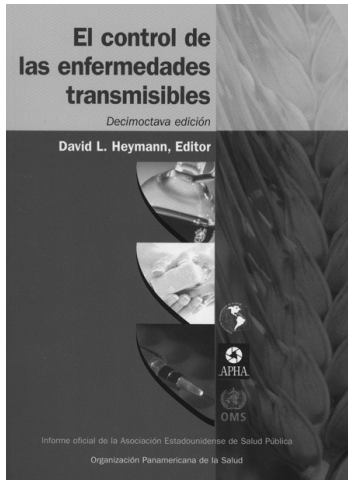

2005, $848 \mathrm{pp}$.,

ISBN 9275316139

Código: PC 613,

Precio: US\$ 22.00 en

América Latina y el

Caribe/ US $\$ 28.00$ en el

resto del mundo
Al igual que en ediciones anteriores, en la presente se explican las características de las enfermedades transmisibles y sus agentes causales, distribución poblacional, reservorios, modos de transmisión, períodos de incubación y contagio, y métodos de control. También se proporciona información acerca del uso deliberado de agentes biológicos con fines nocivos. La publicación se complementa con un glosario de términos de suma utilidad y un exhaustivo índice de materias.

El control de las enfermedades transmisibles es una fuente de información que apoyará a las naciones en su empeño por brindar un acceso universal y equitativo a la salud.

Adquiera esta publicación por medio de la librería en línea de la OPS: http://publications.paho.org Fax: (301) 206-9789 Correo electrónico: paho@pmds.com• OPS/OMS oficina de país 